\title{
Flora vascular de las montañas de Córdoba (ARGENTINA): CARACTERÍSTICAS Y DISTRIBUCIÓN DE LAS ESPECIES A TRAVÉS DEL GRADIENTE ALTITUDINAL
}

\author{
VAscular Flora in the MOUNTAins of CóRdoba (ARgentina): \\ CHARACTERISTICS AND SPECIES DISTRIBUTION ACROSS THE ELEVATION \\ GRADIENT
}

\author{
Melisa A. Giorgis ${ }^{1 *(\mathbb{D})}$, María V. Palchetii ${ }^{1}$, Rita Morero ${ }^{1}$, Marcelo Cabido ${ }^{1}$, \\ Jorge O. Chiapella ${ }^{1,2}$ y Ana M. Cingolani ${ }^{1}$ (I)

\section{SUMMARY}

1. Instituto Multidisciplinario de Biología Vegetal, CONICET- FCEFyN, Universidad Nacional de Córdoba, Córdoba, Argentina

2. Instituto de Investigaciones en Biodiversidad y Medioambiente (INIBIOMA-CONICET-Universidad Nacional del Comahue), Quintral 1250, R8400FRF Bariloche, Río Negro, Argentina

*mgiorgis@imbiv.unc.edu.ar

Citar este artículo GIORGIS, M. A., M. V. PALCHETII, R. MORERO, M. CABIDO, J. O CHIAPELLA \& A. M. CINGOLANI. 2021. Flora vascular de las montañas de Córdoba (Argentina): características y distribución de las especies a través del gradiente altitudinal. Bol. Soc. Argent. Bot. 56: 327-345.

DOI: https://doi. org/10.31055/1851.2372.v56. n3.30355

Recibido: 18 Sep 2020 Aceptado: 21 Jul 2021

Publicado en línea: 2 Sep 2021

Publicado impreso: 30 Set 2021

Editora: Silvia Lomáscolo (DD

ISSN versión impresa 0373-580X

ISSN versión on-line 1851-2372
Background and aims: Mountains hold a large portion of global biodiversity and provide important benefits to humans. With the aim of providing a key tool for the sustainable management of Córdoba mountain ecosystems, we present an exhaustive list of vascular plants and their altitudinal ranges.

M\&M: We compiled 669 complete floristic surveys and 1178 partial surveys performed from 2005 to 2020.

Results: We record 1069 plant species: 147 non-native, 143 endemic to Argentina, 25 endemic to Córdoba and San Luis and 19 endemic to Córdoba. Most species had their median elevation below 1000 masl. Species turnover up to 1250 masl is slow, but from this elevation onwards it begins to accelerate, and continues in a similar way up to the highest end of the gradient. Out of all recorded species, $46 \%$ are recognized as medicinal, of which, 79 are non-natives, 36 are endemic to Argentina, two are endemic to Córdoba and San Luis and two are exclusive of Córdoba.

Conclusions: The list includes $60 \%$ of the species cited for Córdoba province and $65.5 \%$ of the medicinal flora recorded for this province. The upper end of the mountains, which represents less than $10 \%$ of the area, concentrates the highest proportion and diversity of endemic species in central Argentina. Our results show the importance of these mountains for the conservation and sustainable management of Córdoba biodiversity, and represent a baseline for future studies.

\section{Key Words}

Alien species, Chaco Serrano, conservation, endemism, forest, grassland, invasion, restoration.

\section{RESUMEN}

Introducción y Objetivos: Las montañas concentran gran parte de la biodiversidad del planeta y brindan importantes beneficios al ser humano. Con el objetivo de generar una herramienta clave para el manejo sostenible de los ecosistemas de montaña de Córdoba, nos propusimos presentar una lista exhaustiva de plantas vasculares y sus rangos altitudinales.

M\&M: Recopilamos 669 censos florísticos completos y 1178 inventarios parciales realizados a campo desde 2005 hasta 2020.

Resultados: Registramos 1069 especies de plantas: 147 exóticas, 143 endémicas de Argentina, 25 endémicas de Córdoba y San Luis y 19 endémicas de Córdoba. La mayoría de las especies encuentran su mediana altitudinal por debajo de los 1000 $\mathrm{m}$. El recambio de especies hasta los $1250 \mathrm{msnm}$ es lento, pero a partir de esta altitud comienza a acelerarse, y continúa de forma similar hasta el extremo más alto del gradiente. El $46 \%$ de las especies registradas han sido reportadas como medicinales, de ellas, 79 son exóticas, 36 endémicas de Argentina, dos endémicas de Córdoba y San Luis y dos exclusivas de Córdoba.

Conclusiones: El listado incluye el $60 \%$ de las especies citadas para Córdoba y el $65.5 \%$ de la flora medicinal registrada para la provincia. El extremo superior de las montañas, que representa menos del $10 \%$ de la superficie, concentra la mayor proporción y diversidad de especies endémicas del centro de Argentina. Nuestros resultados muestran la importancia de estas montañas para la conservación y el manejo sostenible de la biodiversidad de Córdoba, y representan una línea de base para futuros estudios.

\section{Palabras Claves}

Bosque, Chaco Serrano, conservación, endemismos, exóticas, especies invasoras, pastizales, restauración. 


\section{INTRODUCCIÓN}

Las montañas son paisajes característicos del planeta que cubren una superficie reducida, no obstante, son desproporcionadamente importantes por la cantidad de especies que contienen y porque brindan servicios ecosistémicos esenciales para el bienestar de las personas (Körner, 2007; Rahbek et al., 2019; Mengist et al., 2020; Perrigo et al., 2020). Cambios rápidos en las características ambientales a través del gradiente altitudinal, sumados a la alta rugosidad de las montañas, son la causa de la enorme riqueza que contienen y de los cambios en el ensamble de especies (Körner, 2007; Rahbek et al., 2019). Al mismo tiempo, las partes altas de las montañas constituyen islas biogeográficas rodeadas por una matriz de tierras bajas con características e historias biogeográficas muy diferentes, por lo que suelen concentrar un gran número de especies endémicas que les confieren mayor valor de conservación (Lomolino et al., 2016). Por lo tanto, es muy importante tener listados actualizados y completos de las especies que viven en áreas montañosas.

Durante las últimas décadas, el desarrollo de plataformas digitales que incluyen materiales de herbario, tanto nacionales (p.ej. www.darwin.edu. ar) como internacionales (p.ej. www.theplantlist. org), ha brindado nuevas oportunidades para el acceso a la información florística. Sin embargo, hasta el momento, este tipo de plataformas no permite obtener fácilmente la lista de especies para una región o área en particular. Por otro lado, los cambios constantes en la nomenclatura botánica contribuyen a que la información propia de cada taxón, como así también el registro de sus localidades, se encuentren frecuentemente desactualizados. A esto se suma que los registros suelen estar asociados a caminos y lugares de fácil acceso, sesgando aún más la información sobre la distribución de los taxones, especialmente en el caso de especies raras con distribuciones reducidas. Por estas razones, la utilización de plataformas digitales nacionales o internacionales es muchas veces inapropiada para la reconstrucción de la distribución de las especies a escalas detalladas. En este sentido, las publicaciones de listados de especies provenientes de inventarios florísticos de campo hechos a escalas locales o regionales, resultan de gran relevancia (Biurrun et al., 2019).
Para diseñar y ejecutar cualquier programa de conservación, restauración o uso sostenible de los recursos naturales, es esencial no solo contar con la lista de especies sino, además, disponer de información básica sobre su frecuencia en el área y su distribución geográfica. Por ejemplo, la frecuencia de aparición y la extensión del área de distribución son factores clave para determinar la probabilidad de extinción de las especies y establecer su status de conservación (IUCN, 2019). Es ampliamente reconocido que taxones con una frecuencia baja y/o área de distribución pequeñas, tienen mayores probabilidades de extinción que taxones con características opuestas (p.ej. Gaston, 1998; Pimm et al., 2014). Recientemente, se ha reportado que la tasa de extinción actual de las especies de plantas con semillas es 500 veces mayor que la extinción de fondo (Humphreys et al., 2019). Sin embargo, el mismo estudio también advierte que el $35 \%$ de las especies que aparecen como extintas en las bases de datos son posteriormente redescubiertas. Por estas y otras razones, hay una necesidad imperiosa de aumentar los esfuerzos por conocer y monitorear la frecuencia de los taxones en general $\mathrm{y}$, en especial, en regiones menos estudiadas, como en el caso de numerosos ecosistemas del Neotrópico (Biurrum et al., 2019).

La provincia de Córdoba se ubica en el centro de la República Argentina, en el extremo austral de América del Sur. En el sector oeste de la provincia se extiende un sistema de montañas pertenecientes a las Sierras Pampeanas (Carignano et al., 2014). La composición florística de estas montañas es muy heterogénea y su variación está fuertemente asociada al gradiente altitudinal (Cabido et al., 1991; Acosta et al., 1992; Cantero et al., 2001, 2011; Giorgis et al., 2017; Oyarzabal et al., 2018; Arana et al., 2021). Este gradiente no solo involucra cambios en la temperatura y las precipitaciones, sino también en la incidencia de fuegos (Argañaraz et al., 2020), herbivoría (Argibay \& Renison, 2018; Marcora et al., 2018) y en el estado de avance de la mayoría de las especies exóticas invasoras desde zonas bajas y centros poblados (Giorgis et al., 2011a, 2016).

Además de proveer bienes y recursos como fibras, alimentos o combustible (Díaz et al., 2018), las plantas tienen numerosos usos medicinales. Cerca del $33 \%$ de la flora nativa de Córdoba 


\section{A. Giorgis et al. - Flora vascular de las montañas de Córdoba}

tiene aplicaciones medicinales, siendo la quinta provincia argentina con mayor cantidad de especies con este tipo de usos (Barboza et al., 2009). Numerosos trabajos botánicos y etnobotánicos han abordado esta temática en las últimas décadas (p.ej. Barboza et al., 2006; Arias Toledo et al., 2010; Martínez, 2010; Furlan et al., 2011; Luján et al., 2017). Barboza et al. (2006, 2009) informaron el número aproximado de especies medicinales presentes en toda la provincia de Córdoba, sin discriminar cuáles están en la zona serrana. El resto de los trabajos (por ej. Arias Toledo et al., 2010; Martínez, 2010; Furlan et al., 2011; Luján et al., 2017) ofrecen listados de especies medicinales, pero solo de algunos sectores de las montañas. Consecuentemente, para las montañas de Córdoba, no se conoce aún con precisión cuál es el número total de especies medicinales, ni dónde, ni con qué frecuencia se presentan, información que es esencial para planificar estrategias de manejo y conservación de las mismas.

Las montañas de Córdoba cuentan con listados $\mathrm{y}$ análisis de la flora para algunos sectores o ambientes particulares (por. ej. Cabido et al., 1991; Cantero et al., 2001, 2011, 2021; Cingolani et al., 2003; Giorgis et al., 2011b, 2017; Oggero \& Arana, 2012). En el trabajo de Giorgis et al. (2011b), publicamos por primera vez un listado de plantas vasculares de toda la extensión de las montañas de Córdoba, pero sólo hasta los $1750 \mathrm{msnm}$, excluyendo las zonas más altas. El trabajo fue y sigue siendo muy consultado por distintas instituciones y personas que llevan a cabo proyectos de conservación y restauración. Por ello, en esta nueva contribución proponemos extender y actualizar ese listado a la totalidad de la superficie de las montañas de la provincia, incluyendo el gradiente altitudinal completo y describiendo la distribución de los taxones a través de dicho gradiente. Específicamente, nos planteamos los siguientes objetivos: 1) Proveer el listado de las especies de plantas vasculares que hemos registrado a campo en todas las montañas de Córdoba, entre los años 2005 y 2020. 2) Describir el rango de variación altitudinal de las especies de plantas vasculares registradas, con énfasis en especies leñosas (nativas y exóticas), y endémicas. 3) Identificar las especies medicinales y establecer su representatividad dentro de la flora estudiada.

\section{MÉtodos}

\section{Área de estudio}

El estudio abarca la totalidad del área montañosa de la provincia de Córdoba (aprox. $33700 \mathrm{~km}^{2}$ ), que se conoce genéricamente como Sierras de Córdoba. Estas montañas se componen de tres cordones principales, que abarcan una extensión de $430 \mathrm{~km}$ de norte a sur y $110 \mathrm{~km}$ de este a oeste. Varían altitudinalmente desde aproximadamente 280 a $2790 \mathrm{msnm}$ y a lo largo del gradiente altitudinal la superficie disponible para la biota se reduce considerablemente (Fig. 1 A-C).

El paisaje de estas montañas se encuentra dominado por un complejo mosaico de diferentes fisonomías y ensambles florísticos que varían a través del gradiente altitudinal y de los gradientes geográficos. Estas variaciones, además de asociarse con cambios en los factores ambientales, también se relacionan con la presencia de disturbios antropogénicos y con la invasión de especies exóticas leñosas (Cingolani et al., 2004, 2008; Giorgis et al. 2011a, 2016; Tecco et al., 2016; Argañaraz et al., 2020). Este último proceso, generalmente se desencadena en las zonas más bajas y con el tiempo se expande hacia mayores elevaciones (Giorgis et al., 2011a, 2016, 2017; Tecco et al., 2016). El gradiente altitudinal ha sido dividido, por diferentes autores, en dos sectores con diferentes afinidades biogeográficas (Kurtz, 1904; Nores \& Yzurieta, 1983; Cabido et al., 1998, 2018; Martínez et al., 2017; Arana et al., 2021). Estos autores coinciden en que la historia biogeográfica de los taxones de la parte inferior del gradiente altitudinal estaría relacionada con la Provincia Fitogeográfica Chaqueña, mientras que la historia de los taxones del sector superior estaría más vinculada con el sector andino. Sin embargo, hay disidencias en relación al límite altitudinal a partir del cual se separarían estas dos historias biogeográficas, y diferencias en las denominaciones asignadas a los distintos pisos altitudinales. Por tal motivo, y a los fines de describir el gradiente altitudinal, en esta contribución aplicamos un criterio propio resultante de la combinación de la información de las distintas fuentes consultadas. De este modo, dividimos al gradiente en dos pisos principales (piso Serrano y piso Subandino) $\mathrm{y}$ un piso intermedio de transición entre ellos. El piso Serrano se localiza en los sectores más 
A

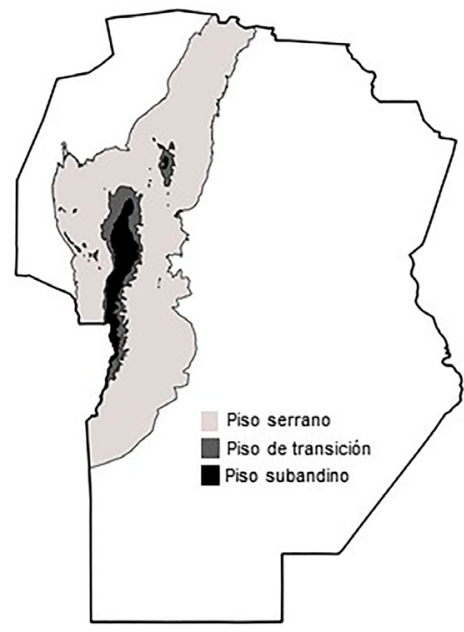

B

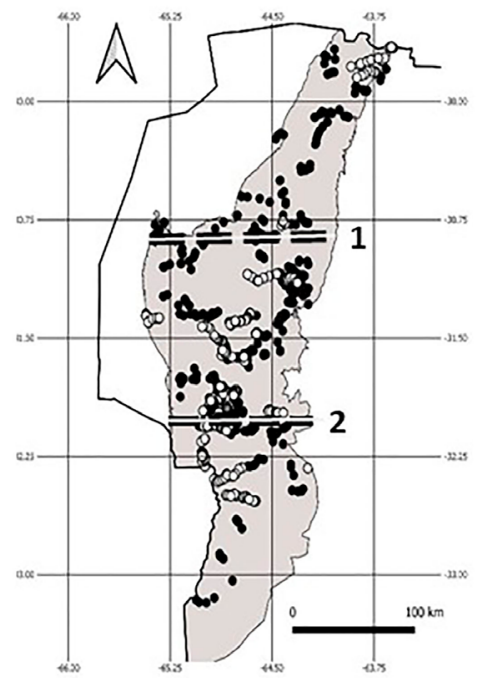

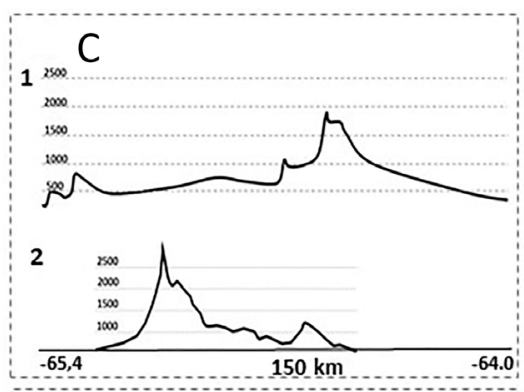

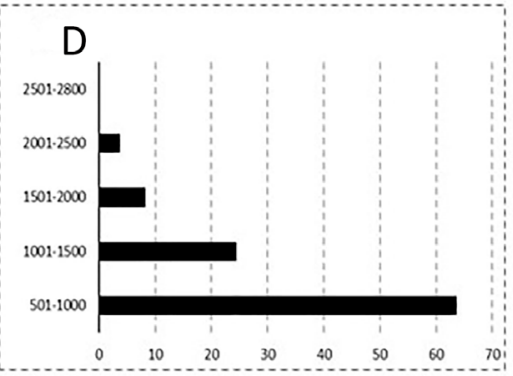

Fig. 1. A: Localización de las sierras en la provincia de Córdoba. B: Ubicación de los sitios de muestreo realizados entre los años 2005 y 2020: los círculos negros indican censos florísticos completos y los blancos inventarios parciales. C: Perfiles altitudinales simplificados de las sierras de este a oeste, a la altura del Cerro Uritorco (1: Lat. $-30,84^{\circ}$ ) y Cerro Champaquí (2: Lat. $\left.-31,98^{\circ}\right)$. D: Área (\%) aproximada que ocupan los diferentes pisos altitudinales de las sierras con respecto a su superficie total $\left(33700 \mathrm{~km}^{2}\right)$.

bajos, llegando hasta aproximadamente los 1300 msnm, límite a partir de cual comienzan a abundar en el paisaje las comunidades dominadas por Festuca spp., y se reduce substancialmente la presencia de comunidades dominadas por Lithraea molleoides y Celtis tala (Giorgis et al., 2017). El piso Subandino está localizado en el sector superior, aproximadamente a partir de los 1700 msnm, coincidiendo aproximadamente con el propuesto por los trabajos de Kurtz (1904) y Cabido et al. (1998). Por arriba de este último límite, son prominentes en el paisaje las comunidades dominadas por Poa stuckertii, Deyeuxia hieronymi y/o Lachemilla pinnata, así como por la especie leñosa Polylepis australis (Cabido \& Acosta, 1985, 1986; Cingolani et al., 2003; Cabido et al., 2018). Entre ambos pisos existe un ecotono complejo que denominamos "piso de transición", siguiendo a Argañaraz et al. (2020).

Las temperaturas decrecen a medida que aumenta la altitud. En promedio, considerando la extensión total de las montañas, la temperatura media anual en el piso Serrano es de $16.5{ }^{\circ} \mathrm{C}$, mientras que en el piso de transición y Subandino es de 13 y
$9.5{ }^{\circ} \mathrm{C}$, respectivamente (Argañaraz et al., 2020). Además, la temperatura disminuye levemente con el aumento de la latitud. En el extremo norte del área de estudio (aprox. $29^{\circ} \mathrm{S}$ ), a $700 \mathrm{~m}$ de altitud, la temperatura media anual es de aproximadamente $17.3^{\circ} \mathrm{C}$, mientras que en el extremo sur (aprox. $32^{\circ} \mathrm{S}$ ), a la misma altitud, es de alrededor de 15.6 ${ }^{\circ} \mathrm{C}$ (Hijmans et al., 2005). En las zonas más bajas, las precipitaciones decrecen del sureste al noroeste (desde $>700 \mathrm{~mm}$ hasta $<500 \mathrm{~mm}$ anuales) (Hijmans et al., 2005), pero también aumentan con la altitud, al menos para algunos sectores en los que hay registros, hasta cerca de los $900 \mathrm{~mm}$ anuales en las partes más altas (Colladon, 2018; Argañaraz et al., 2020).

\section{Obtención de datos}

La fuente de datos consistió en 1847 inventarios de campo que llevamos a cabo entre diciembre del 2005 y marzo del 2020 en todo el territorio de las sierras (Fig. 1B). Del total de inventarios, 669 corresponden a censos florísticos completos (la mayoría incluidos en la base sPlot SA2, en DarkDivNet y, en menor medida, datos inéditos 


\section{A. Giorgis et al. - Flora vascular de las montañas de Córdoba}

de Giorgis et al.); los restantes corresponden a listas parciales de especies de plantas vasculares que consideramos de importancia y han sido tomados por Giorgis et al. (2011b) y Cingolani et al. (datos inéditos). En cada uno de los inventarios completos se registró la totalidad de especies de plantas vasculares presentes (para más detalles ver metodología en Giorgis et al., 2017), mientras que en los inventarios parciales solo se registró la presencia de algunas especies leñosas (ver más detalles en Giorgis et al., 2011b) o bien de especies representativas de las distintas comunidades vegetales de las montañas de Córdoba (Cabido \& Acosta, 1985; Cabido et al., 1991; Cingolani et al., 2003; Giorgis et al., 2017). En conjunto, la distribución de los inventarios florísticos completos y parciales cubre todo el gradiente altitudinal, latitudinal y longitudinal de las sierras de córdoba (Fig. 1B). Es importante aclarar que los costados de caminos y zonas ribereñas, en su mayoría, no están incluidas en estos inventarios.

La pertenencia de las especies a sus respectivas familias y la actualización de los nombres científicos se llevó a cabo siguiendo el «Catálogo de las Plantas Vasculares del Cono Sur» (Zuloaga et al., 2008, 2019) y su actualización «on line» al 10 de marzo de 2020 (www.darwin.edu.ar). En los pocos casos en que no se siguió ese catálogo, se encuentra un comentario asociado a la especie en el Anexo 1. En los casos donde no fue posible asignar un nombre específico, los taxones se nombran a nivel de género (p.ej. Hydrocotyle spp.) $\mathrm{y}$, seguidamente en el Anexo, se listan las especies cuya distribución coincide con el área de estudio (p.ej. H. bonariensis, H. modesta). En el caso de taxones infraespecíficos cabe aclarar que, si el nombre aceptado es una variedad de la especie y si las variedades eran difíciles de discriminar, sólo se las menciona cuando es la única citada para la provincia de Córdoba; contrariamente, cuando hay más de una variedad reportada para la provincia, decidimos no considerar el rango varietal, e indicar únicamente el nombre de la especie.

\section{Categorización de las especies}

Clasificamos las especies según su origen en nativas o exóticas, esta última subdividida en dos categorías: exóticas para Argentina y exóticas para las montañas del centro de Argentina, pero nativas a nivel nacional. En la primera categoría, incluimos a todas aquellas especies que son citadas como adventicias, introducidas, naturalizadas o exóticas para Argentina en el catálogo de las Plantas Vasculares del Cono Sur (Zuloaga et al., 2008, 2019 y sus actualizaciones on-line). En la segunda categoría, siguiendo el criterio de Richardson \& Rejmánek (2011), incluimos a las especies que son nativas de diferentes regiones de Argentina, excepto las montañas del centro del país (montañas de San Luis y Córdoba y las sierras de Sumampa y Ambargasta de Santiago del Estero). Estas dos categorías de exóticas se distinguen en el Anexo 1, pero para los análisis de datos ambos grupos están incluidos dentro de una única categoría de exóticas, ya que solo ocho especies se clasificaron como exóticas para el área de estudio siendo nativas de Argentina. Para llevar a cabo esta clasificación según el origen consultamos las descripciones de cada especie en las diferentes floras disponibles. También revisamos los registros de herbarios disponibles en Documenta Florae Australis (www.darwin.edu.ar/ iris) en los casos de especies para las cuales resultó dificultoso establecer su origen (p.ej. Lantana camara, Schinus areira, entre otras).

Además, clasificamos a las especies nativas según su distribución en dos categorías: endémicas de la Argentina y no endémicas. Por otro lado, y dado que un importante número de taxones son exclusivos de las provincias de Córdoba y San Luis (Giorgis et al., 2011b; Chiapella \& Demaio, 2015), consideramos otras dos categorías de endemismos excluyentes entre sí: endémicas exclusivas de Córdoba y endémicas de Córdoba y San Luis. Para esto, consultamos tanto al catálogo de la Flora del Cono Sur como diversas publicaciones de la Flora Argentina, floras provinciales y trabajos específicos de los diferentes taxones incluidos en nuestra lista.

También clasificamos a las especies según su forma de vida siguiendo parcialmente a Giorgis et al. (2011b) en: árboles, arbustos, cactáceas columnares, cactáceas globulares, cactáceas opuntioides, hierbas latifoliadas perennes (de aquí en más "hierbas perennes" por simplicidad), hierbas latifoliadas anuales (de aquí en más "hierbas anuales"), epífitas, helechos, enredaderas, parásitas, gramíneas en mata y graminoides. Las dos últimas categorías incluyen a los taxones de Poaceae, Cyperaceae, Juncaceae y otras 
monocotiledóneas de hojas finas. En la categoría de "gramíneas en mata" quedaron incorporadas aquellas gramíneas con macollos grandes y robustos, mientras que en la categoría de graminoides quedaron incorporadas las especies con macollos pequeños o no macollantes. Para la clasificación en formas de vida se consideró la forma predominante en el área de estudio, pero es importante aclarar que el hábito anual o perenne de las hierbas o la diferencia entre gramíneas en mata o graminoides puede variar según las condiciones climáticas o la presión de pastoreo.

Finalmente, clasificamos a las especies según si cuentan o no con un registro de uso medicinal. Para esto utilizamos como base las publicaciones de Barboza et al. (2006; 2009). Adicionalmente, utilizamos la información de trabajos publicados en revistas científicas desde el año 2006. Para esto último, realizamos una búsqueda en el sitio web Google Académico (www.scholar.google.com) con las siguientes palabras: Córdoba, Argentina, plant*, medicina*, etnobotan*, farmac*, tradic* el día 1/12/2019. En el Anexo 1, se presenta la bibliografía consultada sobre las especies con usos medicinales.

\section{Análisis de Datos}

Para cada especie con más de tres registros, estimamos la mediana y los valores máximos y mínimos de la altitud en la que estaban ubicados los inventarios que las incluían. Calculamos estos mismos parámetros para la latitud y la longitud. Las especies que aparecieron menos de tres veces sólo quedaron mencionadas en las tablas, pero no se incluyeron en los análisis sobre la distribución geográfica. Además, calculamos la frecuencia relativa para cada una de las especies presentes en los censos florísticos completos $(\mathrm{N}=669)$.

Para describir los patrones emergentes de la distribución de especies en el gradiente altitudinal, graficamos las medianas altitudinales de todas las especies, ordenadas desde aquella que tuvo su mediana a menor altitud hasta la que la tuvo a mayor altitud. Asimismo, distinguimos en estos gráficos a las especies por su forma de vida, endemismo y uso medicinal. Además, para los árboles nativos, las leñosas exóticas, las especies endémicas de Córdoba y San Luis y las exclusivas de Córdoba, más frecuentes, graficamos la distribución altitudinal utilizando gráficos de caja.

\section{Resultados}

\section{Descripción general de la flora}

Registramos 1069 especies en las montañas de Córdoba, que pertenecen a 115 familias y 544 géneros. La curva de acumulación de especies (Anexo 2) sugiere que, en conjunto, nuestros inventarios representaron una alta proporción de la flora de Córdoba. Del total de especies registradas, 147 $(13,7 \%)$ fueron exóticas. Entre estas últimas, ocho fueron nativas a nivel nacional. Las familias más representadas fueron Poaceae (172), Asteraceae (164) y Fabaceae (84) (Anexo 2. Tabla 2.1). Las familias que presentaron mayor número de especies exóticas fueron Asteraceae (23), Poaceae (19) y Rosaceae (14) (Anexo 2. Tabla 2.1). Los géneros con mayor número de especies fueron Euphorbia (16), Baccharis (14), Solanum (14), Nasella (12) y Tillandsia (12) (Anexo 2. Tabla 2.2). Las formas de vida mejor representadas en el conjunto de especies de las montañas de Córdoba fueron las hierbas perennes (365), seguidas por graminoides (169) y por arbustos (139) (Anexo 2. Tabla 2.3). Registramos un total de 143 especies endémicas de Argentina, siendo 25 de ellas endémicas de San Luis y Córdoba y 19 exclusivas de Córdoba. Además, aportamos un nuevo registro para Córdoba, la Orchidaceae Cyclopogon apricus (Lindl.) Schltr.

En el Anexo 1 presentamos la lista completa de especies y, para aquellas especies con tres o más registros en la base de datos (834), también incluimos el rango y la mediana de la altitud, latitud y longitud a las que se encontraron dichas especies.

Las especies nativas más frecuentes, registradas en más del $50 \%$ de los censos florísticos completos, fueron Bidens subalternans, Vachellia caven y Commelina erecta. La especie exótica más frecuente, registrada en el $24 \%$ de los censos completos, fue Talinum paniculatum, seguida por Ligustrum lucidum (15\%) y Cynodon dactylon (14\%).

\section{Nueva cita para Córdoba \\ Cyclopogon apricus (Lindl.) Schltr.}

Hierba perenne, terrestre, de $8-20 \mathrm{~cm}$ de altura. Raíces fasciculadas, carnosas y breves. Hojas pequeñas, 10-30 $\mathrm{mm}$ por 5-10 mm, arrosetadas, de contorno oval-lanceolado, 1-5 nervadas. Escapo constituido por 1-2 hojas caulinares, pubescente-glandulosas, oval-lanceoladas, con el extremo acuminado. Flores (3-35) reunidas en inflorescencias de 10-30 $\mathrm{mm}$ de largo, dispuestas perpendicularmente al raquis, sépalos 
con densa cobertura de pelos glandulosos, brácteas mayores que el largo del ovario.

Hábitat y distribución geográfica. Se encuentra en Brasil, Uruguay y NO y E de la Argentina. En Córdoba se encontró entre los 1520 y1868 msnm, en coberturas de césped y pastizales con roca expuesta por erosión.

ARGENTINA. Prov. Córdoba: Dpto. Río Cuarto: En el extremo superior de las sierras de los Comechingones en el camino que conecta Luti con Pueblo Escondido, 32 ${ }^{\circ} 24^{\prime} 12,5^{\prime \prime} \mathrm{S}, 6^{\circ}$ 54' 36.7" W, 2094 msnm, Giorgis 2006, 22-12-2020 (CORD).

\section{El gradiente altitudinal}

Las especies nativas con mayores rangos altitudinales, que abarcaron más de $2000 \mathrm{~m}$, fueron Tillandsia capillaris (280-2610 msnm), Galium richardianum (367-2693 $\mathrm{msnm})$ y Cardionema ramosissima (367-2693 msnm). Dentro de las exóticas, las especies con mayor rango altitudinal, abarcando más de 1700 m, fueron Taraxacum officinalis (642$2684 \mathrm{msnm})$, Spergula ramosa (475-2245 msnm) y Cirsium vulgare (651-2356 msnm). Las especies con rangos pequeños, de solo $20 \mathrm{~m}$ de variación fueron Phleum alpinum (2589-2609 msnm), Synandrospadix vermitoxicus (615-635 msnm) y Juncus stipulatus (2589-2609 msnm), entre otras (Anexo 1).

La gran mayoría de las especies de las montañas de Córdoba tuvieron medianas a menos de $1000 \mathrm{~m}$ de altitud, comenzando a partir de allí un recambio paulatino de taxones hasta llegar a un punto de inflexión alrededor de los $1250 \mathrm{~m}$. Por arriba de esta altitud el recambio de especies se acelera y se mantiene hasta el extremo superior del gradiente (Fig. 2). Tabebuia nodosa y Arenaria serpens fueron las especies con la menor y mayor mediana altitudinal, respectivamente ( $416 \mathrm{~m}$ y $2676 \mathrm{~m}$ ).
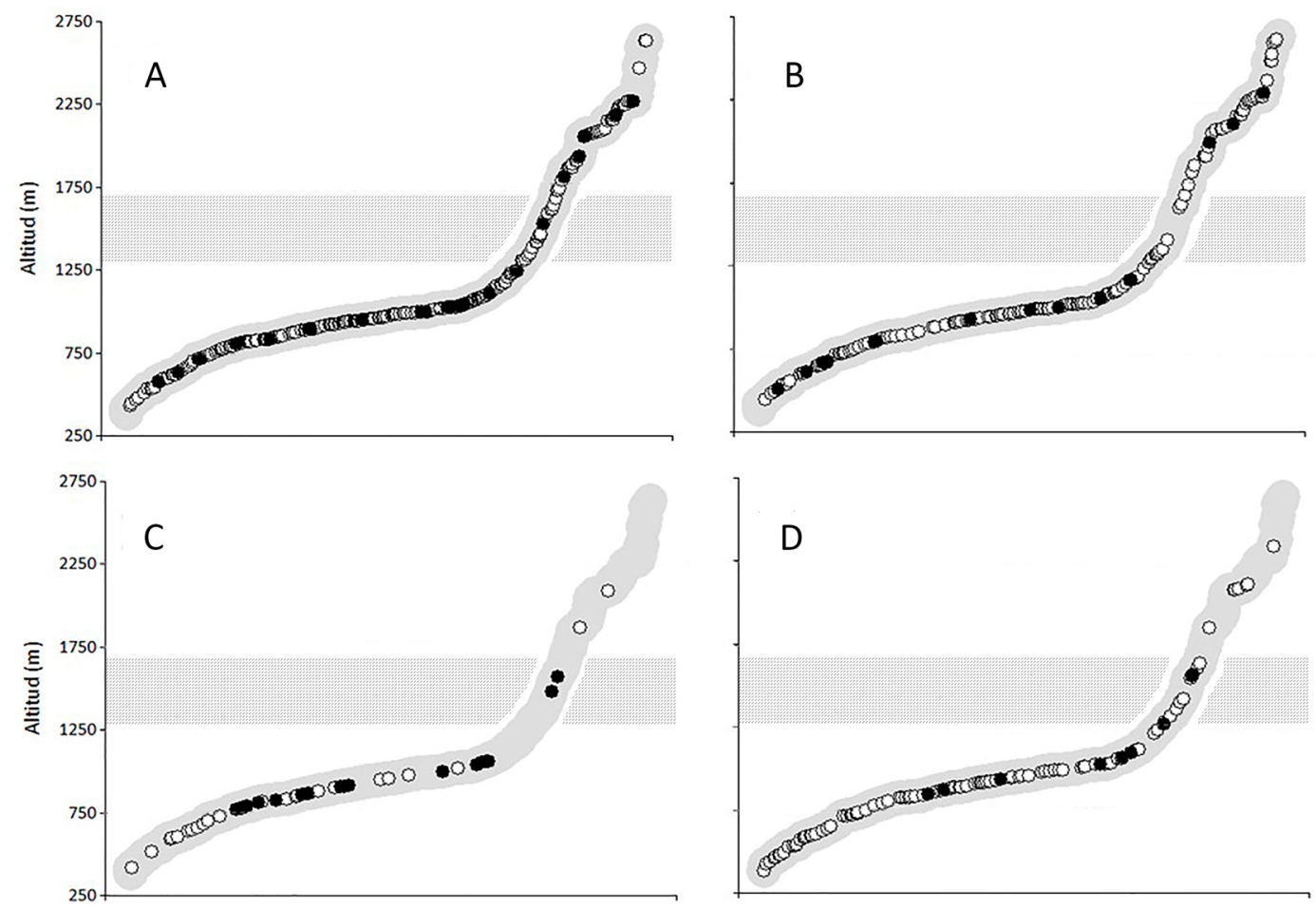

Fig. 2. Medianas altitudinales (identificadas por el sombreado gris rodeando los círculos) de las especies de plantas registradas entre los años 2005 y 2020, con más de tres datos de presencia en las montañas de Córdoba y ordenadas de menor a mayor altitud (ver lista de especies y medianas altitudinales en el Anexo 1). Los círculos blancos representan a las especies nativas y los círculos negros a las exóticas, correspondientes a distintas formas de vida: A: hierbas perennes; B: gramíneas y graminoides; C: árboles; D: arbustos. La franja horizontal gris representa el piso de transición entre los 1300 y $1700 \mathrm{msnm}$. 
Las hierbas perennes, gramíneas y graminoides, tanto nativas como exóticas, siguieron este patrón general (Fig. 2 A-B). En el caso de los árboles nativos, el mayor número de especies también tuvo medianas altitudinales a menos de 1000 m (Figs. 2C, 3). De este grupo, Zanthoxylum coco y Lithraea molleoides tuvieron máximos altitudinales a los 1543 y $1611 \mathrm{~m}$, respectivamente (Fig. 3). Por arriba de éstos y con medianas altitudinales superiores a $1700 \mathrm{~m}$ se encontraron Maytenus boaria y Polylepis australis, cuyos mínimos altitudinales se registraron a 878 y $1397 \mathrm{~m}$, respectivamente (Fig. 3). Los arbustos nativos se distribuyeron de forma continua a lo largo del gradiente. Si bien la mayoría de ellos tuvo medianas altitudinales por debajo de los $1300 \mathrm{~m}$, un pequeño grupo presentó medianas alrededor de los 2000 m de altitud, y una especie aislada (Senecio retanensis), una mediana altitudinal de 2336 m (Fig. 2D).

En el caso de los árboles exóticos, la mayoría presentaron medianas altitudinales entre $\operatorname{los} 750 \mathrm{y}$ $1060 \mathrm{~m}$, mientras que los taxones Betula pendula y Salix spp. tuvieron medianas altitudinales alrededor de los $1500 \mathrm{~m}$ (Fig. 2C). De los árboles exóticos más frecuentes en las montañas, el género Salix se registró a la mayor altitud y tuvo el rango más amplio de variación (990 - $2250 \mathrm{~m})$, seguido hacia abajo en el gradiente altitudinal por Ulmus pumila y Morus alba, quienes también presentaron un rango amplio de variación (Fig. 4). Los arbustos exóticos tuvieron medianas que variaron entre 786 y $1268 \mathrm{~m}$ de altitud (Fig. 2D) y la mayoría de ellos no superó los $1400 \mathrm{~m}$. Las excepciones fueron Cotoneaster franchetii,

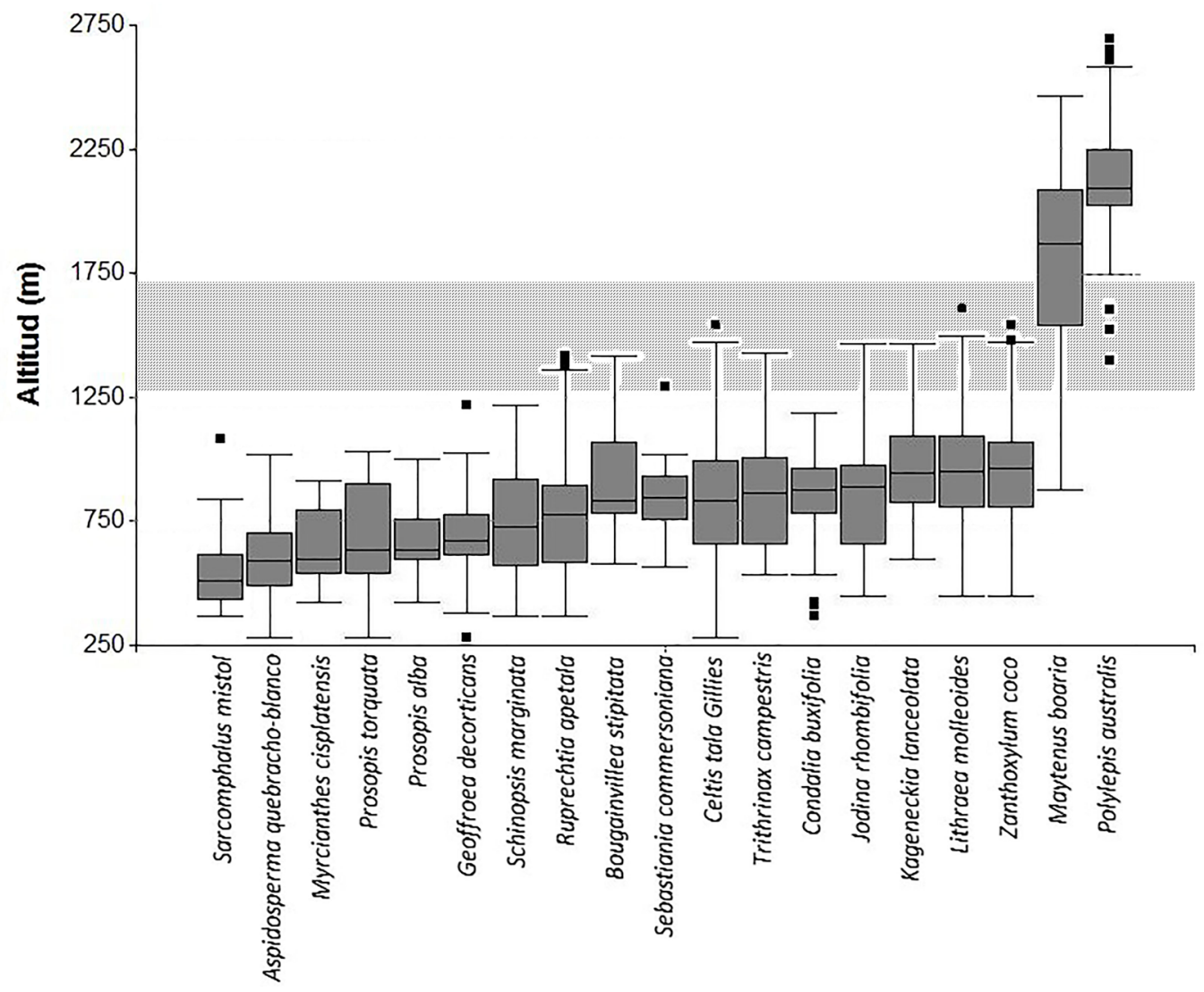

Fig. 3. Diagramas de caja de la distribución altitudinal de los árboles nativos con más de 34 datos de presencia, registrados entre los años 2005 y 2020 en las montañas de Córdoba, en orden creciente según su mediana altitudinal. La franja horizontal gris representa el piso de transición entre los 1300 y $1700 \mathrm{msnm}$. 


\section{A. Giorgis et al. - Flora vascular de las montañas de Córdoba}

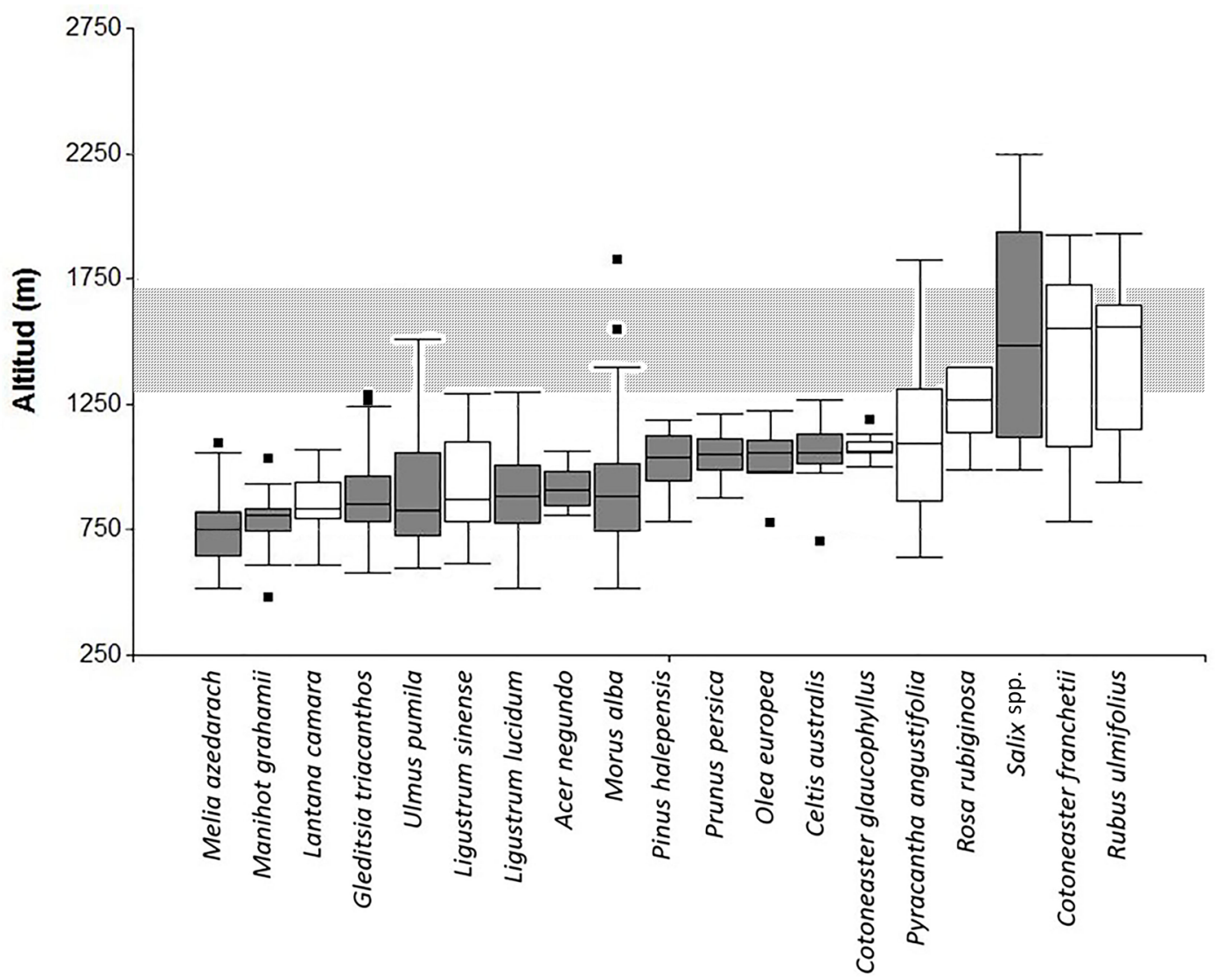

Fig. 4. Diagramas de caja de la distribución altitudinal de los árboles exóticos (gris oscuro) y arbustos exóticos (blanco) con más de 6 datos de presencia, registrados entre los años 2005 y 2020 en las montañas de Córdoba, en orden creciente según su mediana altitudinal. La franja horizontal gris representa el piso de transición entre los 1300 y 1700 msnm.

Rubus ulmifolius y Rosa spp. que se encontraron a altitudes máximas de 1925, 1931 y 1962 m, respectivamente (Fig. 4, Anexo 1).

Las especies endémicas de Córdoba y San Luis estuvieron presentes a través de todo el gradiente altitudinal (Fig. 5). Ocho especies se encontraron casi exclusivamente en el piso Serrano, superando solo muy ocasionalmente los $1300 \mathrm{~m}$. Estas especies se van reemplazando en el gradiente, comenzando desde Acanthocalycium spiniflorum, con una mediana de $493 \mathrm{~m}$, hasta Gymnocalycium bruchii con una mediana de 1221 $\mathrm{m}$. Gymnocalycium monvillei tuvo una mediana a $1256 \mathrm{~m}$ y su rango altitudinal fue muy amplio, abarcando los tres pisos altitudinales, desde los 660 a 2374 m (Fig. 5). Tres especies (Hieracium cordobense, Escallonia cordobensis y Valeriana stuckertii) se encontraron concentradas en el piso de transición, con rangos altitudinales que variaron entre los 1200 y los $1900 \mathrm{~m}$. Finalmente, en el piso Subandino, encontramos nueve especies que raramente crecen por debajo de $\operatorname{los} 1550 \mathrm{~m}$ de altitud. La mediana altitudinal varió desde $2000 \mathrm{~m}$ para Blumenbachia hieronymi hasta $2390 \mathrm{~m}$ para Senecio retanensis, que representa la especie endémica de Córdoba y San Luis cuya distribución alcanza la máxima altitud en el gradiente.

Las especies endémicas exclusivas de Córdoba exhiben un patrón similar a los endemismos de Córdoba y San Luis, ya que se distribuyen a través de todo el gradiente altitudinal (Fig. 6). Encontramos cinco cactáceas globulares del género Gymnocalycium en el 


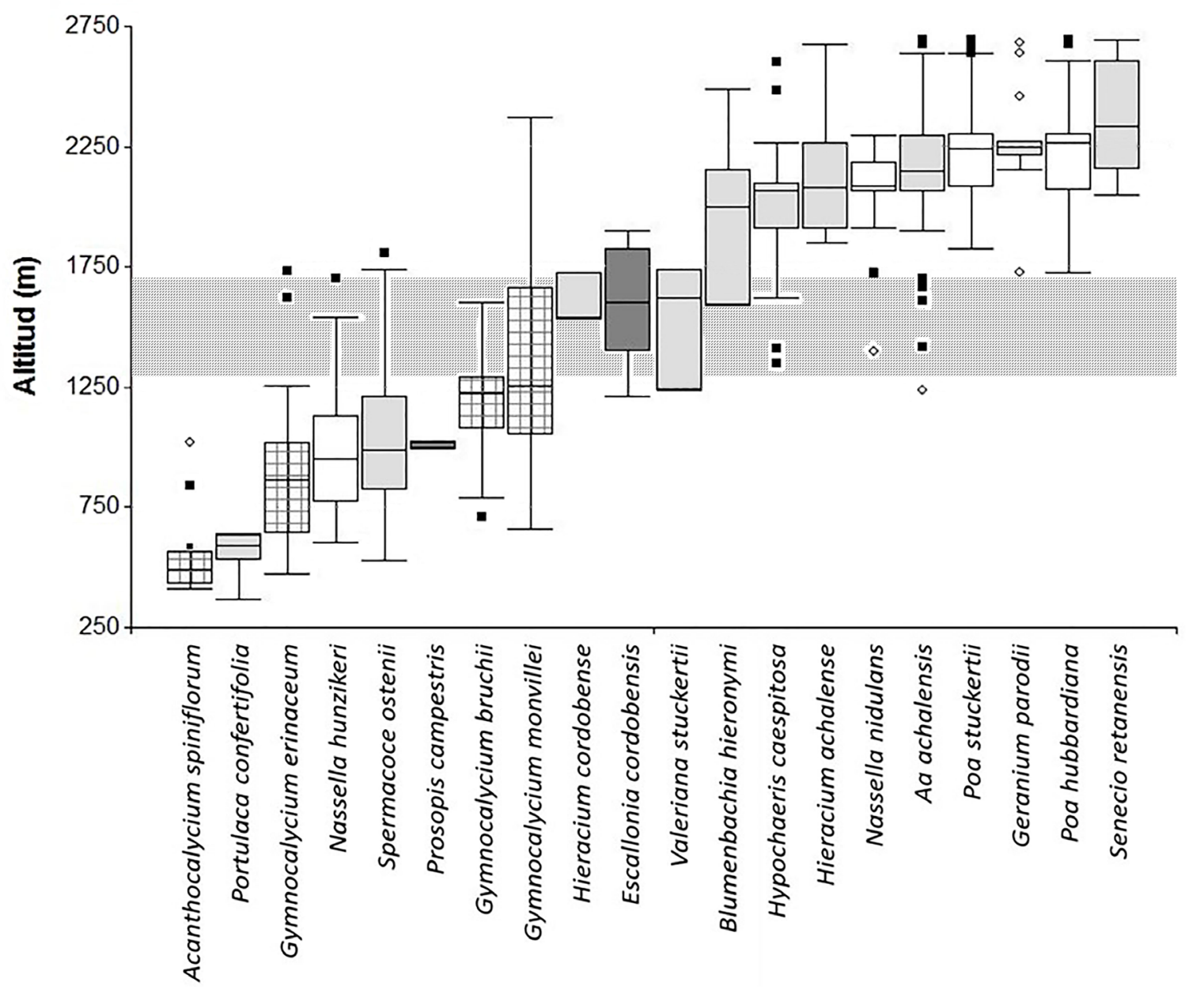

Fig. 5. Diagramas de caja de la distribución altitudinal de las especies endémicas de Córdoba y San Luis con más de tres registros, en orden creciente según su mediana altitudinal. En gris oscuro: arbustos; gris claro: hierbas anuales y perenes; blanco: gramíneas y cuadriculado: cactáceas. La franja horizontal gris representa el piso de transición entre los 1300 y 1700 msnm.

piso Serrano, distribuidas escalonadamente en el gradiente, desde G. castellanosii ssp. ferocius con una mediana altitudinal de $425 \mathrm{~m}$ de altitud hasta $G$. capillense con una mediana altitudinal de $1008 \mathrm{~m}$. Alternanthera pumila tiene una mediana en el piso de transición, a $1307 \mathrm{~m}$, pero su rango altitudinal es muy amplio, abarcando los tres pisos altitudinales. Apurimacia dolichocarpa está restringida al piso de transición, encontrándose en una estrecha franja altitudinal, entre $1400 \mathrm{y}$ $1700 \mathrm{~m}$. En el piso Subandino encontramos siete taxones con rangos altitudinales estrechos y con una mediana altitudinal que varía entre $2112 \mathrm{~m}$ para Gymnocalycium andreae hasta $2633 \mathrm{~m}$ para Hysterionica dianthifolia, siendo esta última la especie endémica de Córdoba que se distribuye a mayor altitud.

\section{Especies con usos medicinales}

El 46\% (496) de las especies registradas en las montañas de Córdoba se reconocen como medicinales. De ellas, el 35\% (175) pertenecen solo a cuatro familias: Asteraceae (97), Fabaceae (37), Euphorbiaceae (21) y Poaceae (20). Asimismo, Verbenaceae y Pteridaceae se destacan ya que el $80 \%$ de sus 21 y 20 especies, respectivamente, 


\section{A. Giorgis et al. - Flora vascular de las montañas de Córdoba}

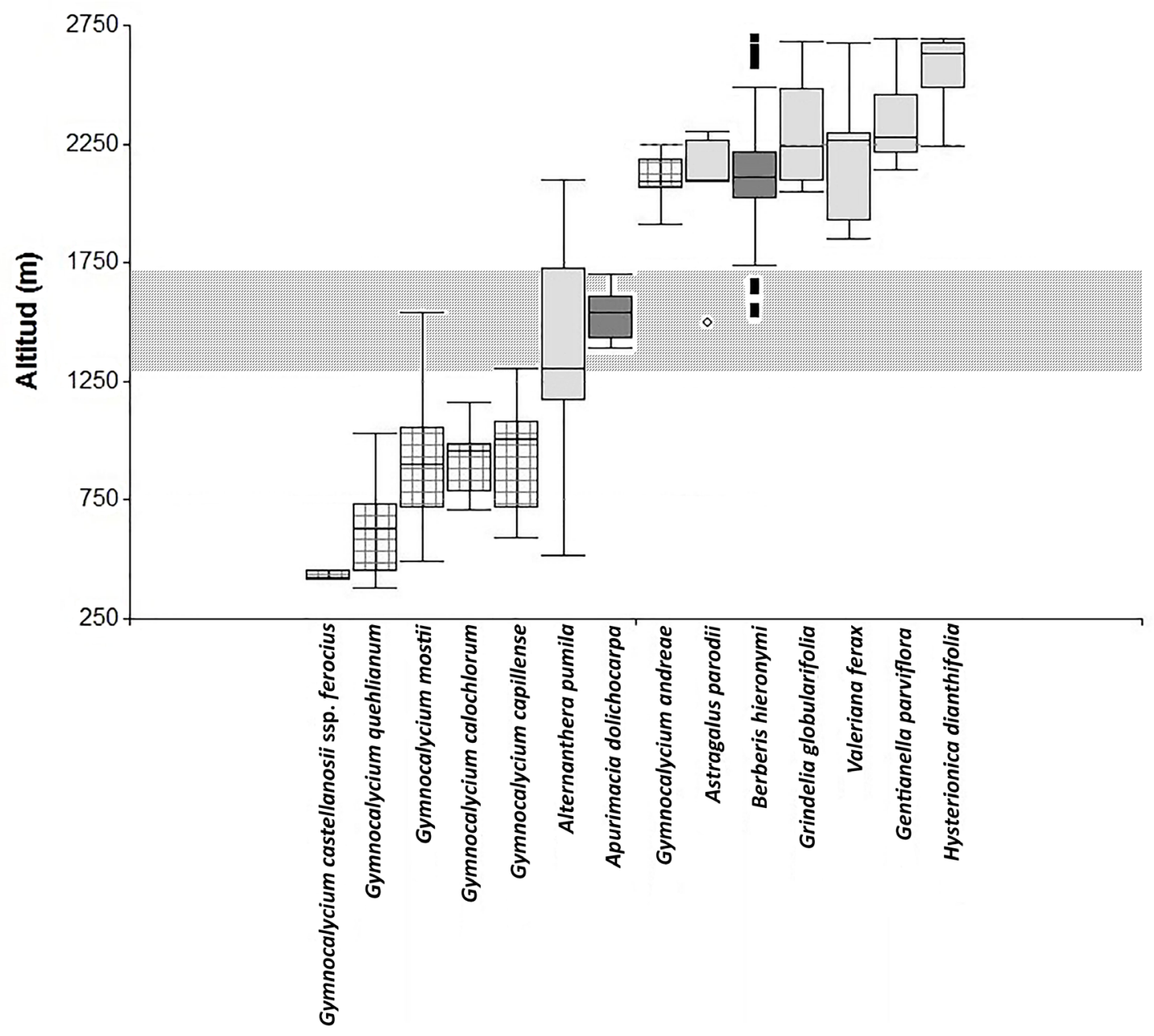

Fig. 6. Diagramas de caja de la distribución altitudinal de las especies endémicas de Córdoba con más de tres datos de presencia, registradas entre los años 2005 y 2020, en orden creciente según su mediana altitudinal. En gris oscuro: arbustos; gris claro: hierbas y cuadriculado: cactáceas. La franja horizontal gris representa el piso de transición entre los 1300 y 1700 msnm.

poseen usos medicinales (Anexo 2, Tabla 2.4). Las formas de vida más representadas dentro de las especies medicinales fueron las hierbas perennes (166 especies), los arbustos (99) y las hierbas anuales (64) (Anexo 2, Tabla 2.4). De las especies con uso medicinal, 79 fueron exóticas, 36 endémicas de Argentina, dos endémicas de Córdoba y San Luis (Trichocline plicata y Sophora linearifolia) y dos endémicas exclusivas de Córdoba (Gentianella parviflora y Valeriana ferax).

La distribución altitudinal de las especies medicinales y no medicinales es relativamente homogénea dentro de las hierbas perennes (Fig. 7A). Las pocas especies de gramíneas y graminoides que tienen este tipo de uso se distribuyen aisladamente en casi todo el gradiente (Fig. 7B). Dentro de los árboles, las especies medicinales, al igual que las no medicinales, concentran sus medianas altitudinales en el piso Serrano. Sin embargo, en el caso de las primeras, hay cuatro que se localizan por encima del piso Serrano, con sus medianas a más de $1400 \mathrm{~m}$ : dos nativas (Maytenus boaria y Polylepis australis) y dos exóticas (Salix spp. y Betula pendula). 

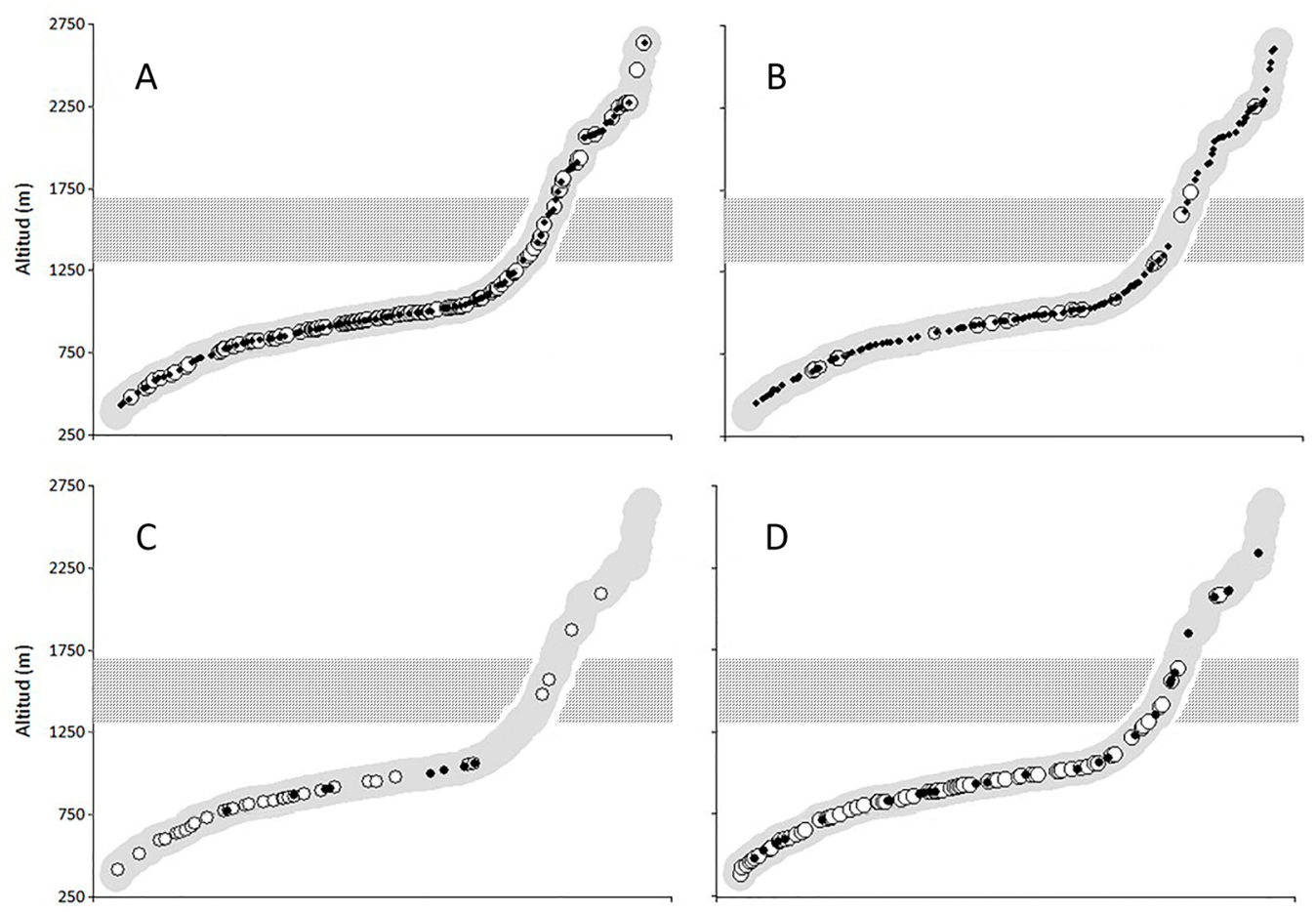

Fig. 7. Medianas altitudinales (identificadas por el sombreado gris rodeando los círculos) de todas las especies de plantas de las montañas de Córdoba, registradas entre los años 2005 y 2020 , que cuentan con más de tres datos de presencia, ordenadas desde la menor a la mayor altitud (ver lista de especies y medianas altitudinales en Anexo 1). En cada gráfico, se destacaron con círculos blancos las especies con uso medicinal y con círculos negros las especies sin uso medicinal, según diferentes formas de vida: A: hierbas perennes; B: gramíneas y graminoides; C: árboles y D: arbustos. La franja horizontal gris representa el piso de transición entre los 1300 y 1700 msnm.

\section{Discusión}

Las montañas del mundo son ecosistemas clave para los seres humanos, ya que contribuyen con servicios y bienes como agua para consumo, energía eléctrica y recreación, entre otros; $y$, además, porque constituyen reservorios fundamentales de la biodiversidad global (Khan et al., 2013; Mengist et al., 2020). En consonancia con estos patrones globales, la lista de plantas vasculares presentada en este trabajo para las montañas de Córdoba incluye cerca del $60 \%$ de las especies citadas para la provincia (Zuloaga et al., 2008) y el $65,5 \%$ de las especies nativas medicinales (Barboza et al., 2009). Estos resultados resaltan la ineludible importancia de realizar un manejo adecuado de estos territorios para garantizar la conservación de la biodiversidad de la provincia.

\section{El gradiente altitudinal}

Encontramos que la mayoría de las especies registradas tiene su mediana altitudinal en el piso Serrano, con un recambio paulatino de especies que se acelera a partir de los $1250 \mathrm{~m}$, hasta llegar al límite superior alrededor de los $2700 \mathrm{~m}$ donde encuentra su mediana Arenaria serpens, la especie que se distribuye a mayor altitud. Este patrón está principalmente determinado por hierbas perennes, gramíneas y graminoides, que son las formas de vida más diversas de la flora de las montañas de Córdoba (Giorgis et al., 2011b). Las medianas altitudinales de la mayoría de los árboles nativos también se ubican en el piso Serrano, por debajo de los $1000 \mathrm{~m}$ de altitud, y sus máximos raramente superan los $1500 \mathrm{~m}$. Recién por arriba de $\operatorname{los} 1700 \mathrm{~m}$ de altitud encuentran su mediana los árboles nativos Maytenus boaria y Polylepis 


\section{A. Giorgis et al. - Flora vascular de las montañas de Córdoba}

australis, que forman los bosques dominados por P. australis en el piso Subandino (Cabido et al., 2018), aunque sus mínimos se extienden hasta el piso de transición e incluso el piso Serrano en el caso de M. boaria. Los arbustos nativos se reemplazan de forma gradual en el gradiente, pero la reducción en su riqueza con la altitud es notoria. En contraposición, los árboles y arbustos exóticos, que constituyen una de las principales amenazas para la flora nativa (Giorgis \& Tecco, 2014), se localizan principalmente entre los 750 y $1750 \mathrm{~m}$, pudiendo llegar incluso hasta casi $2000 \mathrm{~m}$. Si bien estas especies exóticas todavía están limitadas por la presión de propágulos (Giorgis et al., 2011a, 2016), y aún son escasas en el piso Subandino, nuestros resultados sugieren que representan una grave amenaza para las comunidades nativas en todo el gradiente altitudinal, coincidiendo con lo reportado por trabajos anteriores (Giorgis et al., 2011a; Zeballos et al., 2014; Milani et al., 2020). Por otro lado, los resultados indican que hay un recambio en la identidad de las especies exóticas con la altitud. Este recambio está asociado tanto con las características intrínsecas de las especies exóticas (Tecco et al., 2016), como con las prácticas culturales de los habitantes que han condicionado qué especies y en qué cantidad han sido introducidas (Giorgis et al., 2011a; Archibald et al., 2020). Por ejemplo, la presencia de Betula pendula y Salix spp., está relacionada con prácticas culturales de los habitantes de la zona alta de las montañas, así como con características propias de las especies. Puntualmente, Betula pendula está avanzando desde la localidad de La Cumbrecita, localizada aproximadamente a $1400 \mathrm{~m}$ (Giorgis et al., 2011a). Sin embargo, en los últimos años, ya se han registrado individuos reproductivos aislados por arriba de los $1800 \mathrm{~m}$ de altitud muy alejados de su fuente original de propágulos (observación personal). Debido a que $B$. pendula es una especie pionera en bosques boreales (Palmé et al., 2003), podríamos hipotetizar que no existirían restricciones climáticas para el establecimiento y crecimiento de individuos en el extremo superior de las montañas de Córdoba.

Los arbustos exóticos invasores Rosa rubiginosa, Cotoneaster franchetii y Rubus ulmifolius han sido registrados ya en el piso Subandino. $R$. rubiginosa y $R$. ulmifolius son especies espinosas que forman parches monoespecíficos, en altas densidades podrían generar impactos negativos en la producción de carne para consumo. Por otro lado, la expansión de los árboles Pinus radiata y $P$. elliottii en las montañas de Córdoba ha sido relacionada a cambios en la estructura y diversidad de las comunidades (Fiandino et al., 2018), a la reducción local en el número de especies nativas (Giorgis et al., 2005), como así también, a una merma en la disponibilidad de agua para el consumo (Jobbágy et al., 2013) y cambios en la tasa de ciclado de nutrientes (Furey et al., 2014). Recientemente, Milani et al. (2020) mostraron cómo el avance de $P$. radiata y $P$. elliottii constituye una amenaza silenciosa a la dinámica general de los sistemas serranos. Consecuentemente, en línea con los trabajos mencionados, nuestros resultados sugieren que las futuras reglas que definan los ensambles de las comunidades y los servicios que proveen las montañas de Córdoba, estarán condicionadas en parte por el avance de las diferentes especies leñosas exóticas invasoras.

\section{Especies endémicas en el gradiente altitudinal}

En el área de estudio registramos 25 especies endémicas de Córdoba y San Luis y 19 exclusivas de Córdoba que, en conjunto, representan el 69,5\% de las especies endémicas del centro de Argentina reportadas por Chiapella \& Demaio (2015). Del total de especies endémicas registradas, la mayoría (47\%) encuentra su mediana altitudinal en el piso Subandino, seguido por el piso Serrano (38\%) $\mathrm{y}$ el piso de transición (15\%) (ver Fig. 5 y 6). Considerando que el piso Subandino representa menos del $10 \%$ de la superficie de las montañas, la alta concentración de especies endémicas en ese territorio coincide con los resultados de Cabido et al. $(1998,2018)$ y con los patrones macroevolutivos descriptos a nivel global (Körner, 2004; Spehn et al., 2011; Lomolino et al., 2016; Rahbek et al., 2019; Perrigo et al., 2020). Por otro lado, muchas de las especies endémicas que tienen su distribución centrada en el piso Serrano pertenecen al género Gymnocalycium, indicando que la concentración de endemismos está fuertemente relacionada con la historia evolutiva de este género, mientras que las 16 especies propias del piso Subandino (ver Fig. 5 y 6) pertenecen a 15 géneros diferentes, sugiriendo que la historia de aislamiento de este sector ha sido mucho más severa que en los pisos inferiores. Este proceso ha sido señalado anteriormente en 
otros trabajos (Nores \& Yzurieta, 1983; Cabido et al., 1998, 2018; Martínez et al., 2017; Arana et al., 2021).

Finalmente, encontramos sustanciales diferencias en los rangos altitudinales de algunas especies con respecto a los resultados reportados por Chiapella $\&$ Demaio (2015), quienes se basaron en registros de herbario publicados en la base de datos www. darwin.edu.ar. Por ejemplo, Poa stuckertii, Poa hubbardiana, Nassella nidulans, Berberis hieronymi y Geum brevicarpellatum son especies características del piso Subandino y no de las zonas más bajas, mientras que Apurimacia dolichocarpa es una especie endémica propia del piso de transición (Funes \& Cabido, 2008; Grossi \& Funes, 2011), que no se presenta a $3000 \mathrm{~m}$ de altitud como está indicado en la base de datos mencionada. Esto ilustra las limitaciones que puede tener el uso de registros de herbario como única fuente de información, evidenciando, al mismo tiempo, la importancia de contar con inventarios intensivos a campo para describir los rangos altitudinales de las especies.

Representatividad de las especies medicinales en la flora de las montañas

El $27,7 \%$ de las especies nativas de Argentina y el $65,5 \%$ de las especies nativas de Córdoba con usos medicinales (Barboza et al., 2009) se pueden encontrar en las montañas de Córdoba. Estos datos refuerzan la importancia de las montañas como área de conservación de plantas con usos terapéuticos comprobados o en prospección de potenciales fuentes de compuestos bioactivos altamente demandados en la actualidad (Balunas \& Kinghorn, 2005). En coincidencia con Barboza et al. (2009), Asteraceae y Fabaceae son las dos familias con mayor número de especies medicinales en las montañas de Córdoba. Ahora bien, aunque Verbenaceae y Pteridaceae están menos representadas, también se destacan pues el $80 \%$ de sus especies poseen usos medicinales. Verbenaceae incluye numerosas especies medicinales, como aquellas pertenecientes a los géneros Glandularia, Lantana, Lippia y Verbena y especies como Aloysia gratissima (Arias Toledo et al., 2010; Furlan et al., 2011; Trillo \& Audisio, 2018). Los usos reportados para las especies de Verbenaceae son muy variados, pero predominan aquellos relacionados con sus propiedades digestivas y diuréticas. Si bien, muchas de estas especies son frecuentes y características de los ensambles de vegetación en el piso Serrano (Giorgis et al., 2017), los componentes químicos y principios activos de la mayoría de ellas son aún desconocidos (Barboza et al., 2006). Las especies de Pteridaceae son principalmente utilizadas en trastornos del aparato reproductor $\mathrm{y}$, en menor medida, para dolencias relacionadas con los sistemas respiratorio y urinario (Scarpa \& Cassá, 2015). Las especies de esta familia se presentan a lo largo de todas las montañas, pero la distribución y frecuencia es muy variada y característica de cada especie (Morero et al., 2014); por lo tanto, cualquier estudio o prospección de las mismas deberá tener presente este detalle en particular. Adicionalmente, en el listado hay otras especies de helechos y licófitas emblemáticas por sus usos medicinales (p.ej. Phlegmariurus saururus, Birri et al., 2017), por lo que, en conjunto, estos taxones constituyen un recurso medicinal de gran relevancia para esta región.

En este estudio se registraron cuatro especies endémicas del centro de Argentina con usos medicinales; entre ellas, solo Gentianella parviflora ha sido mencionada previamente en la literatura etnobotánica (Luján et al., 2017). Este resultado indica que, en general, las especies endémicas son poco utilizadas y tal vez escasamente conocidas por la población. La reducida mención de los endemismos como medicinales puede deberse a que las dos especies presentes en el piso Serrano, y por lo tanto, más cercanas a centros poblados (Trichocline plicata y Sophora linearifolia), tienen una frecuencia relativa muy baja en el paisaje, mientras que las otras dos especies (Gentianella parviflora y Valeriana ferax) se encuentran restringidas al piso Subandino $y$, por ello, alejadas de las poblaciones donde se han realizado la mayoría de los estudios etnobotánicos en Córdoba.

\section{Sesgos de esta base de datos}

La distribución de los taxones registrados en el presente trabajo corresponde exclusivamente al área estudiada (todas las Sierras de Córdoba). Por lo tanto, cualquier conclusión relacionada a los patrones de distribución de esos taxones, debe quedar limitada a esta área. Además, es importante destacar que como las zonas ribereñas fueron escasamente representadas en los inventarios, especies asociadas a estos ambientes pueden estar ausentes en la lista (p. ej., Amauropelta achalensis, Salix humboldtiana), o bien algunos taxones incluidos en nuestras listas 


\section{A. Giorgis et al. - Flora vascular de las montañas de Córdoba}

pueden tener gradientes geográficos más amplios que los registrados en esta base (p.ej. Polylepis australis, Escallonia cordobensis, Aspidosperma quebracho-blanco). Especies asociadas a bordes de caminos, en particular especies exóticas (Giorgis et al., 2011a, 2016), también pueden estar ausentes en las listas provistas o bien podrían tener gradientes de distribución más amplios. Asimismo, especies endémicas del área con distribución muy restringida pueden estar ausentes en esta base de datos (p.ej. Isostigma cordobense; Mimosa cordobensis, más detalles consultar Chiapella \& Demaio, 2015). Información complementaria sobre la flora de las sierras de Córdoba puede encontrarse en numerosos trabajos publicados, entre los que se destacan las contribuciones de Bianco \& Cantero (1986); Cantero et al. (2001, 2011, 2017, 2021), Oggero \& Arana (2012) y Zeballos et al. (2021).

\section{Consideraciones finales}

Los listados actualizados y fácilmente disponibles de especies de plantas son esenciales para el desarrollo de políticas de manejo, restauración y conservación de los ecosistemas (Humphreys et al., 2019; Roberson et al., 2020). Por lo tanto, al presentar la primera lista de plantas de la totalidad de las montañas de Córdoba, registradas desde el año 2005 hasta la actualidad, pretendemos brindar una herramienta para la conservación y manejo de estos sistemas. Esta base permitirá, entre otros aspectos: 1) seleccionar especies clave para la conservación y restauración de la flora en diferentes sectores de las sierras; 2) evaluar cambios en el tiempo de la frecuencia y distribución de las especies y, por lo tanto, conocer puntualmente cambios en el avance y distribución de las especies exóticas asociados a cambios globales en el clima y en el uso del suelo; 3 ) conocer la frecuencia y distribución de especies con potenciales usos medicinales que, junto con futuros trabajos etnobotánicos, permitirá abordar hipótesis acerca de por qué y cómo seleccionamos las especies para su uso (Gaoue et al., 2017).

\section{Contribución de los Autores}

MAG y AMC escribieron el artículo con contribuciones de todos los autores. MAG, MVP, JOC, MC realizaron la identificación de las especies con contribuciones de AMC Y RM. MAG, MVP y RM realizaron a búsqueda y chequeo de la flora medicinal. MAG, MC, AMC, MVP y RM realizaron los inventarios de campo.

\section{Agradecimientos}

Este trabajo está dedicado al Dr. Luis Ariza Espinar por tantos años de acompañamiento $\mathrm{y}$ aprendizajes brindados. Agradecemos a la enorme cantidad de personas que nos han acompañado a lo largo de todos los años en los numerosos viajes de campo en los que se realizaron los inventarios incluidos en este trabajo. A los siguientes colegas por su colaboración en la identificación de algunas especies: A. A. Coccuci, P. Demaio, L. Carbone, A. Sérsic., G. Barboza, F. Chiarini. A los investigadores y técnicos del Museo Botánico (IMBIV), particularmente a su director, Dr. G. Bernardello; a las asistentes del Herbario: J. Ponce, P. Hick y P. Wiemer. Y finalmente a la editora y a tres revisores anónimos por sus valiosos comentarios y sugerencias. Este trabajo fue financiado por Neotropical Grassland Conservancy, Inter-American Institute for Global Change Research (IAI) CRNII-2005, SECYT, CONICET y MINCyT Córdoba. MAG, JOC, MC y AMC, son investigadores de CONICET. MAG, MVP y RM son profesoras de la UNC.

\section{Bibliografía}

ACOSTA, A., S. DÍAZ, M. MENGHI \& M. CABIDO. 1992. Patrones comunitarios a diferentes escalas espaciales en pastizales de las Sierras de Córdoba, Argentina. Rev. Chil. Hist. Nat. 65: 195-207.

ARANA, M. D, E. NATALE, N. FERRETTI, G. ROMANO, A. OGGERO, G. MARTÍNEZ, P. POSADAS \& J. J. MORRONE. 2021. Esquema biogeográfico de la República Argentina. Opera Lilloana 56: 1-238.

ARCHIBALD, J. L., C. B. ANDERSON, M. DICENTA, C. ROULIER, K. SLUTZ \& E. A. NIELSEN. 2020. The relevance of social imaginaries to understand and manage biological invasions in southern Patagonia. Biol. Invasions 22: 3307-3323. https://doi.org/10.1007/s10530-020-02325-2

ARGAÑARAZ, J. P., A. M. CINGOLANI, L. M. BELLIS \& M. A. GIORGIS. 2020. Fire incidence 
along an elevation gradient in the mountains of central Argentina. Ecol. Austral 30: 268-281.

https://doi.org/10.25260/EA.20.30.2.0.1054

ARGIBAY, D. S. \& D. RENISON. 2018. Efecto del fuego y la ganadería en bosques de Polylepis australis (Rosaceae) a lo largo de un gradiente altitudinal en las montañas del centro de la Argentina. Bosque (Valdivia) 39: 145-150.

https://doi.org/10.4067/S0717-92002018000100145

ARIAS TOLEDO, B., C. TRILLO \& M. GRILLI. 2010. Uso de plantas medicinales en relación al estado de conservación del bosque en Córdoba, Argentina. Ecol. Austral 20: 235-246.

BALUNAS, M. J. \& A. D. KINGHORN. 2005. Drug discovery from medicinal plants. Life Sci. 78: 431441. https://doi.org/10.1016/j.lfs.2005.09.012

BARBOZA, G. E., J. J. CANTERO, C. NUÑEZ, L. ARIZA ESPINAR \& A. PACCIARONI. 2009. Medicinal plants: A general review and a phytochemical and ethnopharmacological screening of the native Argentine Flora. Kurtziana 34:7-365.

BARBOZA, G. E., J. J. CANTERO, C. NUÑEZ \& L. ARIZA ESPINAR (eds.). 2006. Flora medicinal de la Provincia de Córdoba (Argentina): Pteridófitas $y$ Antófitas silvestres o naturalizadas. Museo Botánico de Córdoba, Córdoba, Argentina.

BIANCO, C. \& J. J. CANTERO. 1986. Las plantas vasculares del Suroeste de la Provincia de Córdoba. Catálogo preliminar de las especies. Rev. UNRC 6: 5-52.

BIRRI, M., M. VALLEJO, M. CARRO-JUÁREZ \& A. M. AGNESE. 2017. Aphrodisiac activity of Phlegmariurus saururus in copulating and noncopulating male rats. Phytomedicine 24: 104110. https://doi.org/10.1016/j.phymed.2016.11.020

BIURRUN, I., E. BERGMEIER, J. DENGLER, F. JANSEN \& W. WILLNER. 2019. Vegetation classification and its application are relevant globally. Phytocoenologia 49: 1-6.

https://doi.org/10.1127/phyto/2019/0323

CABIDO, M., \& A. ACOSTA. 1985. Estudio fitosociológico en bosques de Polylepis australis Bitt. (Tabaquillo) en las Sierras de Córdoba, Argentina. Doc. Phytosociol. 9: 385-400.

CABIDO, M. \& A. ACOSTA. 1986. Contribución al conocimiento fitosociológico del Subpiso Superior de Pastizales de las Sierras de Córdoba (Argentina). Veröff. Geobot. Inst. Eidgenöss. Tech. Hochsch., Stift. Rübel Zür 91: 118-140.
CABIDO, M., G. FUNES, E. PUCHETA, F. VENDRAMINI \& S. DÍAZ. 1998. A chorological analysis of the mountains from central Argentina. Is all what we call Sierra Chaco really Chaco? Contribution to the study of the flora and vegetation of the Chaco: XII. Candollea 53: 321-331.

CABIDO, M., M. L. CARRANZA, A. ACOSTA \& S. PÁEZ. 1991. Contribución al conocimiento fitosociológico del Bosque Chaqueño Serrano en la Provincia de Córdoba, Argentina. Phytocoenología 19: 547-566.

https://doi.org/10.1127/phyto/19/1991/547

CABIDO, M., S. R. ZEBALLOS, M. ZAK, M. L. CARRANZA, M. A. GIORGIS, J. J. CANTERO \& A. T. R. ACOSTA. 2018. Native woody vegetation in central Argentina: Classification of Chaco and Espinal forests. Appl. Veg. Sci. 21: 298-311. https://doi.org/10.1111/avsc. 12369

CANTERO, J. J., M. CABIDO, C. NÚÑEZ, L. PETRYNA, M. ZAK \& M. ZOBEL. 2001. Clasificación de los pastizales de suelos sobre rocas metamórficas de las Sierras de Córdoba, Argentina. Kurtziana 29: 27-77.

CANTERO, J. J., J. A. SFRAGULLA, C. NÚÑEZ, A. A. BONALUMI, J. MULKO, A. AMUCHASTEGUI, F. CHIARINI, G. E. BARBOZA \& L. ARIZA ESPINAR. 2011. Flora de los afloramientos de mármoles y serpentinitas de las Sierras de Córdoba (Argentina). Kurtziana 36: 11-45.

CANTERO, J. J., J. MULKO, C. NÚÑEZ, S. R. ZEBAllos, J. A. SFragulla, A. AMUCHASTEGUI, G.E.BARBOZA, F.CHIARINI, L. ARIZA ESPINAR, A. A. BONALUMI \& M. R. CABIDO. 2017. Heterogeneidad de la vegetación en ambientes basálticos del centro de Argentina. Bol. Soc. Argent. Bot. 52: 153-183.

https://doi.org/10.31055/1851.2372.v52.n1.16916

CANTERO, J. J., C. O. NÚÑEZ, S. R. ZEBALLOS, J. SFRAGULLA, A. AMUCHÁstegui, P. BRANDOLIN, A. BONALUMI \& M. R. CABIDO. 2021. Vegetation and flora of marble outcrops and their nearby matrices in mountains of central Argentina. Rock chemistry also matters. Flora 274 : 151757. https://doi.org/10.1016/j.flora.2020.151757

CARIGNANO, C., D. KRÖHLING, S. DEGIOVANNI \& M. CIOCCALE. 2014. Geomorfología. In: MARTINO, R. D. \& A. B. GUERESCHI (eds.), Relatorio del XIX Congreso Geológico Argentino: Geología y Recursos Naturales de la Provincia de Córdoba. pp. 747-822. Asociación Geológica Argentina, Córdoba. 


\section{A. Giorgis et al. - Flora vascular de las montañas de Córdoba}

CHIAPElla, J. O. \& P. H. DEMAIO. 2015. Plant endemism in the Sierras of Córdoba and San Luis (Argentina): understanding links between phylogeny and regional biogeographical patterns. PhytoKeys 47: 59-96. https://doi.org/10.3897/phytokeys.47.8347

CINGOLANI, A. M., D. RENISON, M. R. ZAK \& M. R. CABIDO. 2004. Mapping vegetation in a heterogeneous mountain rangeland using Landsat data: an alternative method to define and classify land-cover units. Rem. Sens. Environ. 15: 84-97. https://doi.org/10.1016/j.rse.2004.05.008

CINGOLANI, A., D. RENISON, P. TECCO, D. E. GURVICH \& M. CABIDO, 2008. Predicting cover types in a mountain range with long evolutionary grazing history: a GIS approach. J. Biogeogr. 35: 538-551.

https://doi.org/10.1111/j.1365-2699.2007.01807.x

CINGOLANI, A. M., M. R. CABIDO, D. RENISON \& V. SOLÍS NEFFA. 2003. Combined effects of environment and grazing on vegetation structure in Argentine granite grasslands. J. Veg. Sci. 14: 223-232. https://doi.org/10.1111/j.1654-1103.2003.tb02147.x

COLLADON, L. 2018. Anuario pluviométrico 2012/132016/17, cuenca del Río San Antonio. Informe interno del CIRSA, Instituto Nacional del Agua.

DÍAZ, S., U. PASCUAL, M. STENSEKE, B. MARTÍNLÓPEZ, R. T. WATSON, Z. MOLNÁR, R. HILL, K. M. A. CHAN, I. A. BASTE, K. A. BRAUMAN, S. POLASKY, A. CHURCH, M. LONSDALE, A. LARIGAUDERIE, P. W. LEADLEY, A. P. E. VAN OUDENHOVEN, F. VAN DER PLAAT, M. SCHRÖTER, S. LAVOREL, Y. AUMEERUDDYTHOMAS, E. BUKVAREVA, K. DAVIES, S. DEMISSEW, G. ERPUL, P. FAILLER, C. A. GUERRA, C. L. HEWITT, H. KEUNE, S. LINDLEY, Y. SHIRAYAMA. 2018. Assessing nature's contributions to people. Science 359: 270272. https://doi.org/10.1126/science.aap8826

FIANDINO, S. I., J. O. PLEVICH, J. C. TARICO, C. NUÑEZ, V. RUSCH \& J. E. GYENGE. 2018. Effects of low-density Pinus elliottii (Slash pine) afforestation on environmental conditions andhh native plant diversity, in the mountains of central Argentina. Appl. Veg. Sci. 21: 442-450.

https://doi.org/10.1111/avsc. 12385

FUNES, G. \& M. CABIDO. 2008. Relaciones florísticas y características regenerativas en Apurimacia dolichocarpa (Fabaceae), especie endémica del centro de Argentina. Phytocoenologia 38: 107-115. https://doi.org/10.1127/0340-269X/2008/0038-0107
FUREY,C.,P.A.TECCO,N.PEREZ-HARGUINDEGUY, M. A. GIORGIS \& M. GROSSI. 2014. The importance of native and exotic plant identity and dominance on decomposition patterns in mountain woodlands of central Argentina. Acta Oecol. 54: 1320. https://doi.org/10.1016/j.actao.2012.12.005

FURLAN, V., C. TORRES \& L. GALETTO. 2011. Conocimiento y utilización de plantas medicinales por pobladores rurales del Bosque Chaqueño Serrano de Córdoba (Argentina). Bonplandia 2: 285-307. https://doi.org/10.30972/bon.2021416

GAOUE, O. G., M. A. COE, M. BOND, G. HART, B. C. SEYLER \& H. MCMILLEN. 2017. Theories and major hypotheses in ethnobotany. Econ. Bot. 71: 269-287.

https://doi.org/10.1007/s12231-017-9389-8

GASTON, K. J. 1998. Species-range size distributions: products of speciation, extinction and transformation. Philos. Trans. R. Soc. London, Ser. B: 353 (1366): 219-230.

https://doi.org/10.1098/rstb.1998.0204

GIORGIS, M. A., A. M. CINGOLANI, D. E. GURVICH, N. REYNERO \& S. RUFINI. 2005. Diferencias en la estructura de la vegetación del sotobosque entre una plantación de Pinus taeda L. (Pinaceae) y un matorral serrano (Cuesta Blanca, Córdoba). Kurtziana 31: 39-49.

GIORGIS, M. A. \& P. A. TECCO. 2014. Invasive alien trees and shrubs in Córdoba province (Argentina): a contribution to the systematization of global bases. Bol. Soc. Argent. Bot. 49: 581-603.

GIORGIS, M. A., A. M. CINGOLANI, D. E. GURVICH, P. A. TECCO, J. CHIAPELLA, F. CHIARINI \& M. CABIDO. 2017. Changes in floristic composition and physiognomy are decoupled along elevation gradients in central Argentina. Appl. Veg. Sci. 20: 558-571. https://doi.org/10.1111/avsc.12324

GIORGIS, M. A., A. M. CINGOLANI, F. CHIARINI, J. CHIAPELLA, G. BARBOZA, L. ARIZA ESPINAR, R. MORERO, D. E. GURVICH, P. A. TECCO, R. SUBILS \& M. CABIDO. 2011b. Composición florística del Bosque Chaqueño Serrano de la provincia de Córdoba, Argentina. Kurtziana 36: $9-43$.

GIORGIS, M. A., A. M. CINGOLANI, P. A. TECCO, M. CABIDO, M. POCA \& H. VON WEHRDEN. 2016. Testing alien plant distribution and habitat invasibility in mountain ecosystems: growth form matters. Biol. invasions 18: 2017-2028. https://oi.org/10.1007/s10530-016-1148-8 
GIORGIS, M. A., P. A. TECCO, A. M. CINGOLANI, D. RENISON, P. MARCORA \& V. PAIARO. 2011a. Factors associated with woody alien species distribution in a newly invaded mountain system of central Argentina. Biol. Invasions 13: 1423-1434. https://doi.org/10.1007/s10530-010-9900-y

GROSSI, M. A. \& G. FUNES. 2011. Biología de especies australes: Apurimacia dolichocarpa (Griseb.) Burkart (Papilionoideae-Leguminosae). Kurtziana 36: 47-52.

HIJMANS, R. J., S. E. CAMERON, J. L. PARRA, P. G. JONES \& A. JARVIS. 2005. Very high resolution interpolated climate surface for global land areas. Int. J. Climat. 25: 1965-1978.

https://doi.org/10.1002/joc. 1276

HUMPHREYS, A. M., R. GOVAERTS, S. Z. FICINSKI, E. N. LUGHADHA \& M. S. VORONTSOVA. 2019. Global dataset shows geography and life form predict modern plant extinction and rediscovery. Nat. Ecol. Evol. 3: 1043-1047.

https://doi.org/10.1038/s41559-019-0906-2

IUCN Standards and Petitions Committee. 2019. Guidelines for Using the IUCN Red List Categories and Criteria. Version 14. Prepared by the Standards and Petitions Committee. Disponible en: http://www. iucnredlist.org/documents/RedListGuidelines.pdf.

JOBBÁGY, E. G., A. ACOSTA \& M. D. NOSETTO. 2013. Rendimiento hídrico en cuencas primarias bajo pastizales y plantaciones de pino de las sierras de Córdoba (Argentina). Ecol. Austral 23: 87-96. https://doi.org/10.25260/EA.13.23.2.0.1164

KHAN, S., S. PAGE, H. AHMAD \& D. HARPER. 2013. Sustainable utilization and conservation of plant biodiversity in montane ecosystems: the western Himalayas as a case study. Ann. Bot. 112: 479-501. https://doi.org/10.1093/aob/mct125

KÖRNER, C. 2007. The use of 'altitude' in ecological research. Trends Ecol. Evol. 22: 569-574. https://doi.org/10.1016/j.tree.2007.09.006

KÖRNER, C. 2004. Mountain biodiversity, its causes and function. $A M B I O$ 33: 11-17.

https://doi.org/10.1007/0044-7447-33.sp13.11

KURTZ, F. 1904. Flora de Córdoba. In: RÍO, M. E. \& L. ACHÁVAL (eds.), Geografía de la Provincia de Córdoba, vol. 1, pp. 270-343. Compañía Sudamericana de Billetes de Banco, Buenos Aires, Argentina.

LOMOLINO, M. V., B. R. RIDDLE \& R. J. WHITTAKER. 2016. Biogeography, pp 1-730. Sinauer Associates, Oxford University Press, Oxford.
LUJÁN, M. C. \& G. J. MARTÍNEZ. 2017. Dinámica del conocimiento etnobotánico en poblaciones urbanas y rurales de Córdoba (Argentina). Bol. latinoam. Caribe plantas med. Aromát. BLACPMA 16: 278-302.

LUJÁN, M. C., G. E. BARBOZA \& G. MARTÍNEZ. 2017. Confluencia de experiencias etnomédicas y uso de plantas medicinales en practicantes nativos del Valle de Traslasierra (Departamento San Javier), Córdoba, Argentina. Bol. Soc. Argent. Bot. 52: 797825 .

https://doi.org/10.31055/1851.2372.v52.n4.18873

MARCORA, P. I., A. E. FERRERAS, S. R. ZEBALLOS, G. FUNES, S. LONGO, C. URCELAY \& P. A. TECCO. 2018. Context-dependent effects of fire and browsing on woody alien invasion in mountain ecosystems. Oecol. 188: 479-490.

https://doi.org/10.1007/s00442-018-4227-y

MARTÍNEZ G. J. 2010. Las plantas en la medicina tradicional de las Sierras de Córdoba: Un recorrido por la cultura campesina de Paravachasca y Calamuchita. Ediciones del Copista, Córdoba, Argentina.

MARTÍNEZ, G. A., M. D. ARANA, A. J. OGGERO \& E. S. NATALE. 2017. Biogeographical relationships and new regionalization of high-altitude grasslands and woodlands of the central Pampean Ranges (Argentina) based on vascular plants and vertebrates. Aust. Syst. Bot. 29: 473-488. https://doi.org/10.1071/SB16046

MENGIST, W., T. SOROMESSA \& G. LEGESE. 2020. Ecosystem services research in mountainous regions: A systematic literature review on current knowledge and research gaps. Sci. Total Environ. 702: 134581. https://doi.org/10.1016/j.scitotenv.2019.134581

MILANI, T., E. G. JOBBÁGY, M. A. NUÑEZ, M. E. FERRERO, G. BALDI \& F. P. TESTE. 2020. Stealth invasions on the rise: rapid long-distance establishment of exotic pines in mountain grasslands of Argentina. Biol. Invasions 22: 2989-3001. https://doi.org/10.1007/s10530-020-02303-8

MORERO, R., M. A. GIORGIS, M. D. ARANA \& G. E. BARBOZA. 2014. Helechos y Licofitas del centro de Argentina. Cultivo y especies ornamentales. Editorial Lux. Córdoba, Argentina.

NORES, M. \& D. YZURIETA. 1983. Especiación en las Sierras Pampeanas de Córdoba y San Luis (Argentina), con descripción de siete nuevas subespecies de aves. El Hornero 12: 88-102.

OGGERO, A. J. \& M. D. ARANA. 2012. Inventario de las plantas vasculares del sur de la zona serrana de 


\section{A. Giorgis et al. - Flora vascular de las montañas de Córdoba}

Córdoba, Argentina. Hoehnea 39: 171-199. https://doi.org/10.1590/S2236-89062012000200002

OYARZABAL, M., J. CLAVIJO, L. OAKLEY, F. BIGANZOLI, P. TOGNETTI, I. BARBERIS, H. M. MATURO, R. ARAGÓN, P. I. CAMPANELLO, D. PRADO, M. OESTERHELD \& R. J. C. LEÓN. 2018. Unidades de vegetación de la Argentina. Ecol. Austral 28: 40-63.

https://doi.org/10.25260/EA.18.28.1.0.399

PALMÉ, A. E., Q. SU, A. RAUTENBERG, F. MANNI \& M. LASCOUX. 2003. Postglacial recolonization and cpDNA variation of silver birch, Betula pendula. Mol. Ecol. 12: 201-212. https://doi.org/10.1046/j.1365-294X.2003.01724.x

PERRIGO, A., C. HOORN \& A. ANTONELLI. 2020. Why mountains matter for biodiversity. J. Biogeogr. 47: 315-325. https://doi.org/10.1111/jbi.13731

PIMM, S. L., C. N, JENKINS, R. ABELL, T. M. BROOKS, J. L. GITTLEMAN, L. N. JOPPA, P. H. RAVEN, C. M. ROBERTS \& J. O. SEXTON. 2014. The biodiversity of species and their rates of extinction, distribution, and protection. Science 344: 1246752. https://doi.org/10.1126/science.1246752

RAHBEK, C., M. K. BORREGAARD, R. K. COLWELL, B. O. DALSGAARD, B. G. HOLT, N. MORUETA-HOLME, D. NOGUES-BRAVO, R. J. WHITTAKER \& J. FJELDSA. 2019. Humboldt's enigma: What causes global patterns of mountain biodiversity? Science 365: 1108-1113.

https://doi.org/10.1126/science.aax0149

RICHARDSON, D. M. \& M. REJMÁNEK. 2011. Trees and shrubs as invasive alien species -a global review. Div. Dis. 17: 788-809. https://doi.org/10.1111/j.1472-4642.2011.00782.x

ROBERSON, E. B., A. FRANCES, K. HAVENS, J. MASCHINSKI, A. MEYER \& L. OTT. 2020. Fund plant conservation to solve biodiversity crisis. Science 367: 258-258. https://doi.org/10.1126/science.aba4360

SCARPA, G. F. \& L. A. CASSÁ. 2015. Etnobotánica de los helechos (Ophioglossidae, Equisetidae y Polypodiidae) en Argentina: recopilación y análisis entre grupos criollos e indígenas. Rev. Mus. Argentino Cienc. Nat. 17: 1-12.

https://doi.org/10.22179/REVMACN.17.402

SPEHN, E. M., K. RUDMANN-MAURER \& C. KÖRNER. 2011. Mountain biodiversity. Plant Ecol. \& Div. 4: 301-302. https://doi.org/10.1080/1755087 4.2012.698660

TECCO, P. A., A. I. PAIS BOSCH, G. FUNES, P. MARCORA, S. R. ZEBALLOS, M. CABIDO \& C. URCELAY. 2016. Mountain invasions on the way: are there climatic constraints for the expansion of alien woody species along an elevation gradient in Argentina? J. Plant Ecol. 9: 380-392.

https://doi.org/10.1093/jpe/rtv064

TRILlO, C. \& C. AUDISIO. 2018. Las plantas medicinales de los huertos de pobladores de diferente tradición cultural en Bosques Chaqueños de Córdoba, Argentina. Bol. latinoam. Caribe plantas med. Aromát. 17: 104-119.

ZEBALlOS, S. R., P. A. TECCO, M. CABIDO \& D. E. GURVICH. 2014. Composición de especies leñosas en comunidades invadidas en montañas del centro de Argentina: su relación con factores ambientales locales. Rev. Biol. Trop. 62: 1673-1681.

https://doi.org/10.15517/rbt.v62i4.12995

ZEBALLOS, S. R., M. R. CABIDO, J. J. CANTERO, A. T. ACOSTA, M. V. PALCHETTI. J. ARGARAÑAZ, P. I. MARCORA, P. A. TECCO, A. FERRERAS, G. FUNES, V. M. VAIERETTI, G. CONTI \& M.A. GIORGIS. 2021. Floristic patterns of the neotropical forests, savannas and scrublands with Trithrinax campestris (Arecaceae) in central Argentina. Veg. Classif. Surv. 2: 5. https://doi.org/10.3897/VCS/2021/59384

ZULOAGA, F. O., O. MORRONE \& M. J. BELGRANO (eds.). 2008. Catálogo de las plantas vasculares del Cono Sur (Argentina, sur de Brasil, Chile, Paraguay y Uruguay). Monogr. Missouri Bot. Garden 107: 1-3348.

ZULOAGA, F., M. BELGRANO \& C. ZANOTTI (eds.). 2019. Actualización del Catálogo de las Plantas Vasculares del Cono Sur. Darwiniana N.S. 7: 208-278. https://doi.org/10.14522/darwiniana.2019.72.861

NOTA DEL EDITOR: los anexos de este artículo están disponibles para su descarga como DATOS PRIMARIOS en https://doi.org/10.31055/1851.2372.v56.n3.30355 
